\title{
Cytogenetic Analysis of the Products of Conception After Spontaneous Abortion in the First Trimester
}

\author{
Xueluo Zhang a,b,c Junmei Fan ${ }^{c}$ Yanhua Chen ${ }^{c}$ Jun Wang ${ }^{d}$ Zhijiao Song ${ }^{e}$ \\ Jinghui Zhao ${ }^{c}$ Zhongyun $\mathrm{Li}^{f}$ Xueqing $\mathrm{Wu}^{c}$ Yuanjing Hu${ }^{\mathrm{b}}$

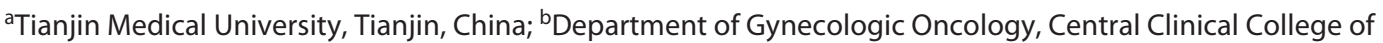 \\ Gynecology and Obstetrics, Tianjin Medical University, Tianjin, China; 'Reproductive Medicine Center, Shanxi \\ Maternal and Child Health Care Hospital, Taiyuan, China; ${ }^{\mathrm{d} D e p a r t m e n t}$ of Orthopedics, Sixth Hospital of Shanxi \\ Medical University (General Hospital of Tisco), Taiyuan, China; 'Department of Prevention and Health Protection, \\ Shanxi Maternal and Child Health Care Hospital, Taiyuan, China; fDepartment of Proctology, Shanxi Provincial \\ Hospital of Traditional Chinese Medicine, Taiyuan, China
}

\section{Keywords}

Early pregnancy loss $\cdot$ Copy number variation $\cdot$ Nextgeneration sequencing $\cdot$ Chromosome $\cdot$ Product of conception

\begin{abstract}
In the present study, we retrospectively recruited 340 patients who underwent spontaneous abortions to investigate chromosomal abnormalities of the conception products in the first trimester. We also performed a relevant analysis of clinical factors. Of these patients, 165 had conception products with chromosomal abnormalities, including 135 aneuploidies, 11 triploidies, 10 complex abnormalities, and 9 segmental aneuploidies. The most common abnormal chromosomes were chromosome 16 in the embryo-transfer group and sex chromosomes in the natural-conception group. The most common abnormal chromosomes in all analyzed maternal age groups were sex chromosomes, 16 , and 22 . The chromosomal abnormality incidence was related to age and number of spontaneous abortions (both $p<0.05$ ), but not to number of pregnancies, deliveries, induced abortions, or methods of conception (all $p>0.05$ ). The rates of abnormal-
\end{abstract}

ity for chromosomes 12, 15, 20, and 22 increased with age, while the rates for chromosomes $6,7,13$, and $X$ decreased. In all age groups, aneuploidy was by far the most common abnormality; however, the low-incidence distributions of chromosomal abnormalities were entirely different. Overall, chromosomal aneuploidy was the primary cause of pregnancy loss in the first trimester, and low-frequency abnormalities differed across age subgroups. Chromosomal aberrations were found to be related to maternal age and spontaneous abortion, but not all chromosomal abnormalities increased with age.

(C) 2021 The Author(s)

Published by S. Karger AG, Basel

\section{Introduction}

Embryo arrest in the first trimester is a type of miscarriage that is defined by a termination of embryonic development due to any reason, and it falls into 2 categories. The first category is defined by conception products

Xueluo Zhang, Junmei Fan, and Yanhua Chen contributed equally to this work.

C 2021 The Author(s)

Published by S. Karger AG, Basel

This article is licensed under the Creative Commons Attribution 4.0 International License (CC BY) (http://www.karger.com/Services/ OpenAccessLicense). Usage, derivative works and distribution are permitted provided that proper credit is given to the author and the original publisher.
Correspondence to:

Yuanjing Hu, julianna_hu@163.com 
that are discharged from the uterus without medical intervention, and the other is a missed abortion where conception products are retained after fetal death. During childbearing age, $25-50 \%$ of women experience miscarriage one or more times, and 10-15\% of pregnancies end in miscarriage. The etiology of miscarriage is complicated, involving heredity, hormones, immunity, uterine morphology, female genital tract infection, semen quality, and environment. Of these, embryonic chromosomal aberration - particularly chromosomal aneuploidy [Sugiura-Ogasawara et al., 2012; Capalbo et al., 2017] - is the dominant risk factor [Rai and Regan, 2006]. Different chromosomal abnormalities produce varied effects on embryonic development [Angiolucci et al., 2011; Ouyang et al., 2016; Zhang et al., 2020], and the frequency of embryonic chromosomal abnormalities is related to clinical factors such as maternal age and the number of previous miscarriages [Ozawa et al., 2019]. With delayed procreation due to changes in personal family planning and population-policy adjustments, the number of women $\geq 37$ years of age planning to conceive has continued to increase, while poor egg quality at this stage of life results in low pregnancy rates and high abortion rates. Assisted reproductive technology (ART) is gradually becoming an important means by which infertile couples become pregnant. Some scientists are concerned, however, because multiple-embryo transfer in ART also implies that some early pregnancy loss may be "hidden" by the continuous growth of companion embryo(s). It is likely that the actual risk of early pregnancy loss would be higher in the population using ART than in the general population [Winter et al., 2002]. Intriguingly, the aneuploidy rate of aborted fetuses after intracytoplasmic sperm injection (ICSI) was significantly higher than after in vitro fertilization (IVF) [Lathi and Milki, 2004]. However, some researchers believe that there are no differences in the incidence of chromosomal aberrations in early miscarriages between conception with ART and natural conception [Miao et al., 2009].

Chromosomal G-banding karyotyping is a popular analysis used to diagnose chromosomal aberrations, but the technique is complicated. First, cell culture is required, which entails a risk of failure due to contamination during the cell-culture process [Shah et al., 2017]. However, with the rapid development of molecular biology, new genetic techniques have gradually emerged including fluorescence in situ hybridization (FISH), array comparative genomic hybridization (array-CGH), single nucleotide polymorphism array (SNP array), and nextgeneration sequencing (NGS).

Chromosomal Abnormalities in Spontaneous Abortions
FISH does not require cell culture (thus avoiding contamination); however, FISH can only detect a few chromosomes at a time. In general, it is used to detect numerical aberrations of chromosomes 13, 16, 18, 21, 22, X, and $\mathrm{Y}$ with specific probes [Jobanputra et al., 2002], and it is difficult to assess all 23 pairs of chromosomes, even with multiple colors and multiple rounds of hybridization. In addition, even with an increasing number of hybridizations, the diagnostic capacity remains diminished. Both SNP and CGH arrays, by contrast, are chip technologies that do not require cell culture. Essentially, the pre-prepared probes are solidified on a chip, and then the isolated DNAs are hybridized with the solidified probes on the chip. Through software analysis, information on the 23 pairs of chromosomes can thus be acquired. However, the diagnostic capacity is limited by the probe source, quality, and density. In addition to the characteristics of the chips, NGS provides high-throughput screening, is rapid, and it is not limited by probes. NGS exhibits a comprehensive open-read capacity for the entire genome and can detect micro copy number variations (CNV) larger than $100 \mathrm{~kb}$. In addition, NGS can detect contaminated and frozen specimens at a lower cost than chip technology [Bauer, 2017]. Thus, NGS is currently widely used in medical diagnostics and genetic disease analysis [Ong et al., 2013].

The population of Shanxi Province primarily consists of local residents, with a small floating population. The majority of the local residents are Han Chinese, with relatively stable population characteristics. For the present study, we retrospectively recruited patients in Shanxi Province who manifested embryo arrest. We investigated the chromosomes of the conception products in the first trimester; summarized the types and distributions of chromosomal aberrations; and analyzed the relationships between chromosomal aberrations and method of conception, age, number of previous miscarriages, pregnancy, delivery, and induced abortion. We then explored the factors related to chromosomal abnormalities of the chorionic villi to provide evidence for genetic counseling of women who underwent spontaneous abortion.

\section{Materials and Methods}

Patients

We recruited 340 patients with early-embryo arrest $(<12$ weeks of gestation) to the Reproductive Medicine Center of Shanxi Maternal and Child Health Care Hospital between January 1, 2016 and July 30, 2019, from couples whose chromosomes were normal. Ultrasonographic diagnostic criteria for early-em-

Cytogenet Genome Res 2021;161:120-13 
Table 1. Details of 165 abnormal chromosomal results

\begin{tabular}{|c|c|c|c|}
\hline Type & Karyotype & Frequency, $n$ & Proportion, $\%$ \\
\hline Triploidy & $69, \mathrm{XNN}$ & 11 & 6.66 \\
\hline \multicolumn{4}{|l|}{ Aneuploidy } \\
\hline \multirow[t]{2}{*}{ Monosomy } & $45, \mathrm{X}$ & 16 & 9.69 \\
\hline & $45, \mathrm{XN},-21$ & 2 & 1.21 \\
\hline \multirow[t]{20}{*}{ Trisomy } & $47, \mathrm{XN},+2$ & 2 & 1.21 \\
\hline & $47, \mathrm{XN},+3$ & 4 & 2.42 \\
\hline & $47, \mathrm{XN},+4$ & 7 & 4.24 \\
\hline & $47, \mathrm{XN},+6$ & 1 & 0.61 \\
\hline & $47, \mathrm{XN},+7$ & 2 & 1.21 \\
\hline & $47, \mathrm{XN},+8$ & 5 & 3.03 \\
\hline & $47, \mathrm{XN},+9$ & 2 & 1.21 \\
\hline & $47, \mathrm{XN},+10$ & 5 & 3.03 \\
\hline & $47, \mathrm{XN},+11$ & 2 & 1.21 \\
\hline & $47, \mathrm{XN},+12$ & 2 & 1.21 \\
\hline & $47, \mathrm{XN},+13$ & 3 & 1.81 \\
\hline & $47, \mathrm{XN},+14$ & 9 & 5.45 \\
\hline & $47, \mathrm{XN},+15$ & 5 & 3.03 \\
\hline & $47, \mathrm{XN},+16$ & 31 & 18.78 \\
\hline & $47, \mathrm{XN},+17$ & 1 & 0.61 \\
\hline & $47, \mathrm{XN},+18$ & 6 & 3.63 \\
\hline & $47, \mathrm{XN},+20$ & 4 & 2.42 \\
\hline & $47, \mathrm{XN},+21$ & 4 & 2.42 \\
\hline & $47, \mathrm{XN},+22$ & 18 & 10.90 \\
\hline & $47, \mathrm{XN},+\mathrm{N}$ & 4 & 2.42 \\
\hline \multirow[t]{10}{*}{ Complex abnormality } & $48, \mathrm{XN},+14,+22$ & 1 & 0.61 \\
\hline & $48, \mathrm{XN},+8,+20$ & 1 & 0.61 \\
\hline & $48, \mathrm{XN},+16,+21$ & 1 & 0.61 \\
\hline & $48, \mathrm{XN},+21,+22$ & 1 & 0.61 \\
\hline & $48, \mathrm{XN},+7,+10$ & 1 & 0.61 \\
\hline & $48, \mathrm{XN},+13,+3$ & 1 & 0.61 \\
\hline & $50, \mathrm{XN},+13,+15,+20,+22$ & 1 & 0.61 \\
\hline & $47, \mathrm{XN},+13, \operatorname{del}(\mathrm{Y})(\mathrm{q} 11.223 \mathrm{q} 11.23), 2.07 \mathrm{Mb}$ & 1 & 0.61 \\
\hline & 47,XN,+7,del(15)(q11.2q21.1), 25.23 Mb & 1 & 0.61 \\
\hline & $46, X,+3$ & 1 & 0.61 \\
\hline \multirow[t]{9}{*}{ Segmental aneuploidy } & 46,XN,del(17)(q12), 1.71 Mb & 1 & 0.61 \\
\hline & 46,XN,del(6)(q24.3q27), 22.95 Mb & 1 & 0.61 \\
\hline & 46,XN,del(3)(p25.2p26.3), 12.57 Mb & 1 & 0.61 \\
\hline & 46,XN,dup(19)(q13.2q13.43), 19.60 Mb & 1 & 0.61 \\
\hline & $46, \mathrm{XN}, \operatorname{dup}(7)(\mathrm{q} 33 \mathrm{q} 36.3), 22.51 \mathrm{Mb}$ & 1 & 0.61 \\
\hline & 46,XN,del(4)(q32.3q35.2), $26.18 \mathrm{Mb}$ & 1 & 0.61 \\
\hline & $46, \mathrm{XN}, \operatorname{del}(1)(\mathrm{q} 42.12 \mathrm{q} 42.3), 8.55 \mathrm{Mb}$ & 1 & 0.61 \\
\hline & 46,XN,del(18)(q21.31q23), 22.29 Mb & 1 & 0.61 \\
\hline & $46, \mathrm{XN}, \operatorname{dup}(1)(\mathrm{p} 36.33 \mathrm{p} 34.1), 45.69 \mathrm{Mb}$ & 1 & 0.61 \\
\hline Total & & 165 & 100.00 \\
\hline
\end{tabular}

Note: Complex abnormality was defined in this study as involving $\geq 2$ chromosomes or 2 types of aberrations.

bryo arrest included (1) crown-rump length $\geq 7 \mathrm{~mm}$ and no heartbeat, (2) mean gestational sac diameter $\geq 25 \mathrm{~mm}$ and no fetal bud, (3) absence of fetal heartbeat $\geq 2$ weeks after the gestational sac emerged without a yolk sac, and (4) absence of fetal heartbeat $\geq 11$ days after the gestational sac showed a yolk sac [Doubilet et al., 2013]. The exclusion criteria included (1) exposure history to toxic and harmful substances during pregnancy, (2) abnormal anatomical structure of the uterus, and (3) serious chronic medical diseases such as severe hypertension or diabetes. When the product of conception was spontaneously discharged from the 
Fig. 1. The constitution of chromosome abnormalities. Overall, 165 patients had conception products with chromosomal abnormalities, including 135 aneuploidies, 11 triploidies, 10 complex abnormalities, and 9 segmental aneuploidies.

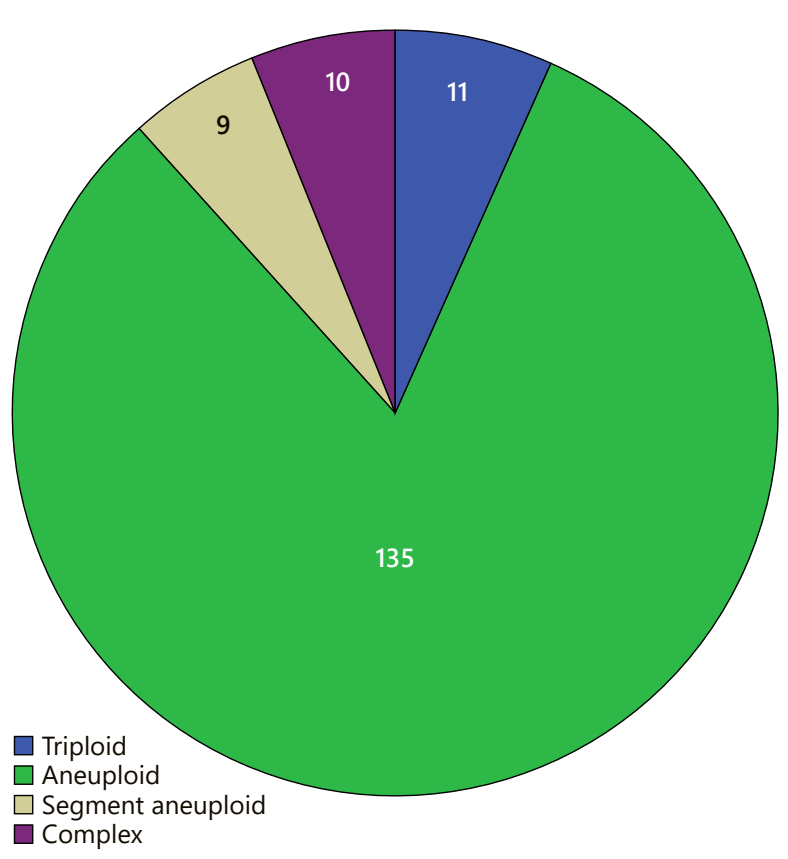

Statistical Analyses

Clinical data are expressed as means \pm 1 standard deviation or median (interquartile range). The Mann-Whitney $U$ test in SPSS 24.0 (IBM, New York) was selected to analyze the differences in clinical factors between normal and abnormal chromosomal groups, and between natural conception and ART conception. We analyzed the disparities in the incidence of chromosomal aberrations between the 2 conception methods using the $\chi^{2}$ test. The differences in aberration rates were compared according to age, pregnancy, delivery, and number of abortions using a $\chi^{2}$ test. $p<0.05$ was considered statistically significant.

\section{Results}

\section{Types and Distribution of Chromosomal}

Abnormalities in Patients with Spontaneous Abortions

A total of 340 patients who underwent spontaneous abortions were included in this study. The average age of the patients was $31.43 \pm 4.18$ years (mean \pm SD, ranging from 23 to 44 years), and the average gravidity was 1.70 \pm 1.12 (range, $1-6$ pregnancies). There were 165 chromosomal abnormalities (48.53\%), and the average age of women in the abnormal group was $32.10 \pm 4.64$ years. The details of the 165 abnormal karyotypes are shown in Table 1 and Figure 1. Aberrations included triploidy $(6.67 \%)$, aneuploidy (81.82\%), complex abnormalities $(6.06 \%)$, and segmental aneuploidies (5.45\%). In addi- 


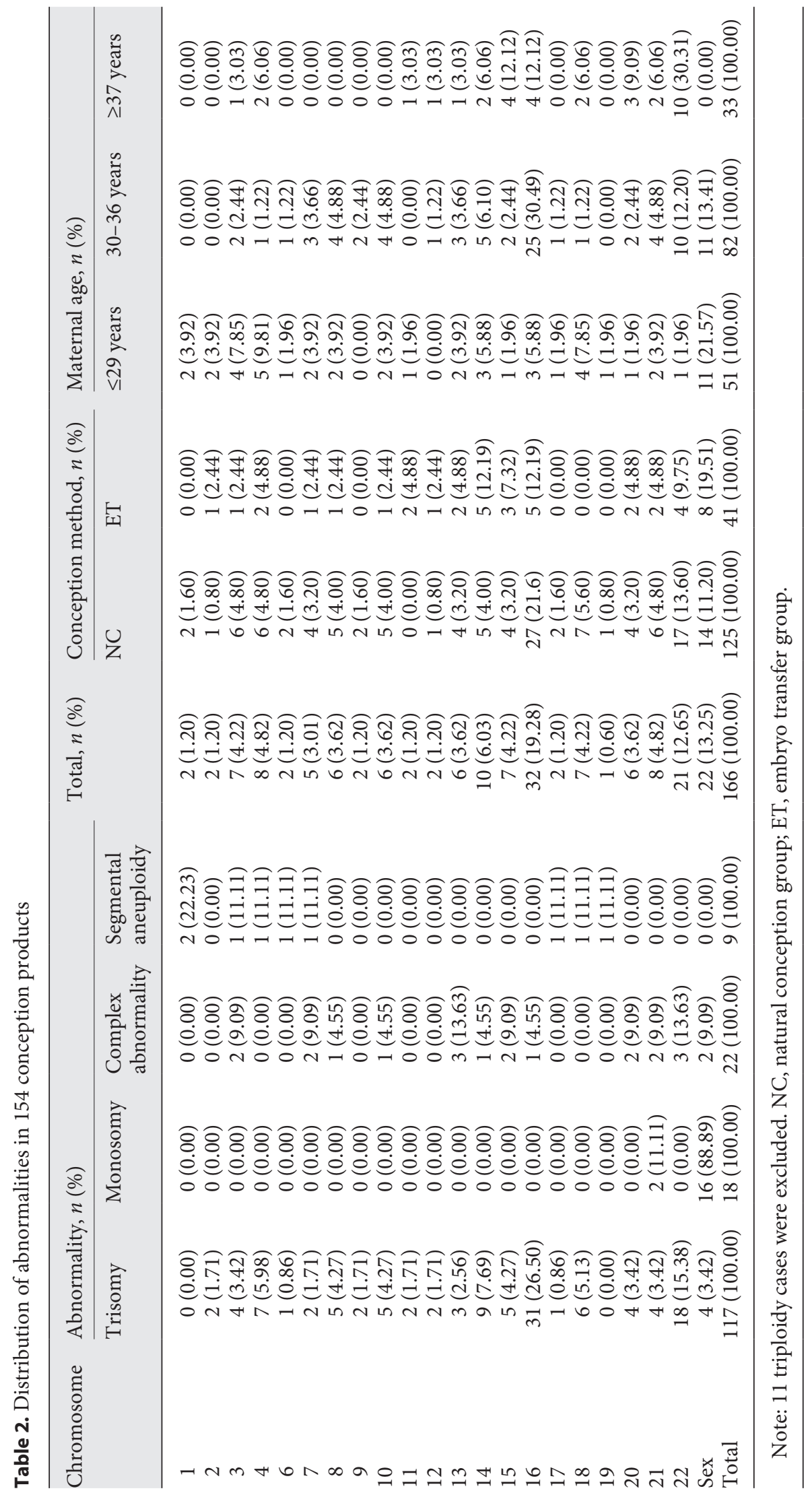




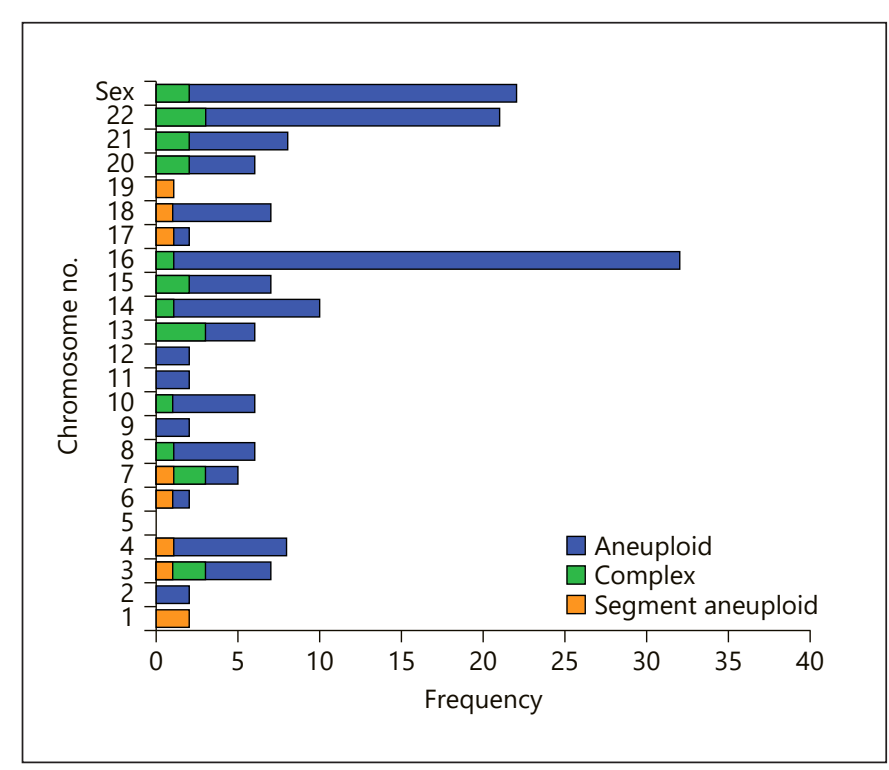

Fig. 2. Chromosomal distribution of abnormalities in conception products. Almost all chromosomes were involved except for chromosome 5. Trisomy 16 and monosomy $\mathrm{X}$ were the major genotypes in the trisomy and monosomy cases, respectively. Monosomy only included chromosomes 21 and X; complex abnormalities primarily affected chromosomes 22 and 13; and segmental aneuploidy principally occurred in chromosome 1 .

tion, a microduplication was detected in a case with complex abnormalities. Among aneuploidy cases, there were 117 with trisomy $(86.67 \%)$ and 18 with monosomy (13.33\%).

Some abnormal cases involved 2 or more chromosomes or 2 or more types of abnormalities, such that 154 abnormal cases, involving 166 abnormal chromosomes, were found after excluding 11 triploid cases. Almost all chromosomes were involved except for chromosome 5. Trisomy 16 and monosomy $\mathrm{X}$ were the major genotypes in trisomy and monosomy cases, respectively. Monosomy only included chromosomes 21 and X; complex abnormalities primarily pertained to chromosomes 22 and 13; and segmental aneuploidy principally occurred in chromosome 1 (Table 2; Fig. 2).

\section{Differences in Chromosomal Abnormalities between \\ the Two Conception Methods}

Based on conception methods, the patients were divided into an embryo-transfer (ET) group (including fresh transfer and frozen-embryo transfer for all individuals participating in ART) and a group with natural conception (NC group). The majority of the chromosomal abnormalities in the NC group involved chromosome 16, while in the ET group, abnormalities primarily occurred in the X chromosome (Table 2). The types of chromosomal abnormalities in the ET and NC groups were different (Table 3). The aneuploidy rate was the highest for anomalies in both the ET and NC groups, and compared to the NC group, the ET group had a lower triploidy rate and no segmental aneuploidies. The rate of complex abnormalities in the NC group was lower than that in the ET group. The risk of chromosomal abnormalities in the ET group was 1.43 times that in the NC group (95\% CI, $0.88-2.32)$, although this was not statistically significant $\left(\chi^{2}=2.143, p>0.05\right)$.

\section{Differences in Chromosomal Abnormalities among Age Groups}

Cases were divided according to maternal age into the following groups: $\leq 29$ years, $30-36$ years, and $\geq 37$ years. Concerning all abnormalities, the rate of aneuploidy was the highest in all 3 age groups. The rate of compound abnormalities was the lowest in the group $\leq 29$ years. The rate of segmental aneuploidies was distinctly lower in the $30-36$ age group than in the $\leq 29$ group, and no segmental aneuploid cases were detected in the group $\geq 37$ years (Table 3). Commensurate with increasing age, the rates for chromosomes 12, 15, 20, and 22 abnormalities increased, while those for chromosomes $6,7,13$, and the sex chromosomes decreased (Table 2). Analysis of the relationship between age and chromosomal abnormalities suggested that abnormalities occurring in early pregnancy were age-related $(p<0.05)$ (Table 4$)$; that is, with increasing age, the risk of chromosomal abnormalities increased in early pregnancy $\left(p^{\mathrm{f}}=0.001 ; \chi^{2}\right.$ trend analysis). There were significant differences between the $\geq 37$ and the $\leq 29$ age groups, and between the $\geq 37$ and $30-36$ age groups $(p<0.013)(\geq 37$ years vs. $\leq 29$ years, OR $=$ $4.20,95 \%$ CI, $1.90-8.92$; $\geq 37$ years vs. $30-36$ years, $\mathrm{OR}=$ $3.02,95 \% \mathrm{CI}, 1.43-6.41)$. The difference was significant between chromosomes from the normal and abnormal groups of conception products in the NC group $(p<$ $0.05)$, and with increasing age, the risk for chromosomal abnormalities in early pregnancy increased $\left(p^{f}=0.001\right)$. The difference was statistically significant between the $\geq 37$ group and the $\leq 29$ group $(p<0.013 ; \mathrm{OR}=4.81,95 \%$ CI, 1.80-12.84). With increasing age in the ET group, the risk of chromosomal abnormalities in early pregnancy increased $\left(p^{\mathrm{f}}=0.048\right)$; however, there was no statistically significant difference with respect to maternal age between normal and abnormal villous chromosomes $(p>$ 0.05; Table 5). 
Table 3. Relationship between clinical factors and abnormal chromosomes

\begin{tabular}{|c|c|c|c|c|c|}
\hline \multirow[t]{2}{*}{ Clinical factor } & \multicolumn{4}{|c|}{ Abnormal embryonic karyotype, $n(\%)$} & \multirow[b]{2}{*}{ Total } \\
\hline & Triploidy & Aneuploidy & $\begin{array}{l}\text { Segmental } \\
\text { aneuploidy }\end{array}$ & $\begin{array}{l}\text { Complex } \\
\text { abnormality }\end{array}$ & \\
\hline \multicolumn{6}{|c|}{ Maternal age, years } \\
\hline$\leq 29$ & $3(5.77)$ & $39(75.00)$ & $8(15.38)$ & $2(3.85)$ & $52(100.00)$ \\
\hline $30-36$ & $6(7.32)$ & $69(84.14)$ & $1(1.22)$ & $6(7.32)$ & $82(100.00)$ \\
\hline$\geq 37$ & $2(6.45)$ & $27(87.10)$ & $0(0.00)$ & $2(6.45)$ & $31(100.00)$ \\
\hline \multicolumn{6}{|c|}{ Spontaneous abortions, $n$} \\
\hline 1 & $2(3.64)$ & $42(76.36)$ & $5(9.09)$ & $6(10.91)$ & $55(100.00)$ \\
\hline 2 & $4(5.97)$ & $57(85.07)$ & $2(2.99)$ & $4(5.97)$ & $67(100.00)$ \\
\hline$\geq 3$ & $5(11.63)$ & $36(83.72)$ & $2(4.65)$ & $0(0.00)$ & $43(100.00)$ \\
\hline \multicolumn{6}{|l|}{ Conception } \\
\hline ET group & $1(2.7)$ & $33(89.19)$ & $0(0.00)$ & $3(8.11)$ & $37(100.00)$ \\
\hline NC group & $10(7.81)$ & $102(79.69)$ & $9(7.03)$ & $7(5.47)$ & $128(100.00)$ \\
\hline
\end{tabular}

ET, embryo transfer; NC, natural cycle.

Table 4. Relationship between clinical factors and chorionic chromosome abnormalities in early pregnancy loss

\begin{tabular}{|c|c|c|c|c|c|c|}
\hline \multirow[t]{2}{*}{ Clinical factor } & \multicolumn{2}{|c|}{ Chromosome result } & \multirow[t]{2}{*}{$p$} & \multicolumn{2}{|c|}{ Conception method } & \multirow[t]{2}{*}{$p$} \\
\hline & Normal & Abnormal & & $\mathrm{NC}$ & ET & \\
\hline Maternal age & $30(4)$ & $31(8)$ & 0.000 & $30(5)$ & $32(6)$ & 0.000 \\
\hline Gravidities & $3(2)$ & $3(1)$ & 0.120 & $3(1)$ & $2(2)$ & 0.130 \\
\hline Deliveries & $0(1)$ & $0(1)$ & 0.272 & $0(1)$ & $0(1)$ & 0.053 \\
\hline Induced abortions & $0(0)$ & $0(0)$ & 0.288 & $0(0)$ & $0(0)$ & 0.597 \\
\hline Spontaneous abortions & $2(1)$ & $2(2)$ & 0.005 & $2(1)$ & $2(2)$ & 0.008 \\
\hline
\end{tabular}

Data are presented as median (interquartile range). ET, embryo transfer; NC, natural cycle.

Differences in Chromosomal Abnormalities Related to Number of Previous Miscarriages

The occurrence of chromosomal abnormalities was related to the number of previous spontaneous abortions ( $p$ $<0.05$ ) and not related to the number of pregnancies, deliveries, or previous induced abortions (all $p>0.05)$ ( Table 4). Based on the number of previous spontaneous abortions, cases were divided into 3 groups: a group with 1 previous spontaneous abortion, a group with 2 previous spontaneous abortions, and a group with $\geq 3$ previous spontaneous abortions. Of the chromosomal abnormalities, aneuploidy showed the highest rate in all subgroups with respect to different prevalences of spontaneous abortion. The triploidy rate was lowest in the group with 1 previous spontaneous abortion, and the segmental aneuploidy rate was lowest in the group with 2 previous spontaneous abortions. No complex abnormality was detected in the group with $\geq 3$ previous spontaneous abortions (Table 3 ).
We observed a significant difference in the number of previous spontaneous abortions between the normal and abnormal groups $(p<0.05)$; that is, with an increasing number of spontaneous abortions per case, the risk of chromosomal abnormalities diminished $\left(p^{\mathrm{f}}=0.002\right)$. There was a significant difference between the groups with 1 versus $\geq 3$ previous spontaneous abortions $(p<0.013$; OR $=0.469$, $95 \%$ CI, 0.27-0.82). In the NC group, the number of previous spontaneous abortions was also significantly different between the normal and abnormal chromosomal groups $(p<0.05)$ : as the number of previous spontaneous abortions increased, the risk of chromosomal abnormalities decreased $\left(p^{\mathrm{f}}=0.001\right)$. This difference was significant between the group with 1 versus $\geq 3$ previous spontaneous abortions ( $p<0.013$; OR $=0.33,95 \% \mathrm{CI}, 0.17-0.66)$. In the ET group, the number of previous spontaneous abortions was not significantly different between the normal and abnormal chromosome groups ( $p>0.05$; Table 6$)$. 
Table 5. Subgroup analysis of chromosomal results in 340 cases as stratified by maternal age

\begin{tabular}{|c|c|c|c|c|c|c|c|}
\hline Group & Age, years & Normal, $n(\%)$ & Abnormal, $n(\%)$ & OR & $95 \% \mathrm{CI}$ & $p$ & $p^{\mathrm{f}}$ \\
\hline \multirow[t]{2}{*}{ Total } & $\leq 29$ & $76(43.43)$ & $52(31.51)$ & 1 & & 0.001 & \multirow[t]{2}{*}{0.001} \\
\hline & $30-36$ & $88(50.29)$ & $82(49.70)$ & 1.362 & $0.86-2.17$ & 0.191 & \\
\hline \multirow[t]{2}{*}{$\mathrm{NC}$} & $\leq 29$ & $59(50.43)$ & $45(35.15)$ & 1 & & 0.004 & \multirow[t]{2}{*}{0.001} \\
\hline & $30-36$ & $52(44.44)$ & $61(47.66)$ & 1.538 & $0.90-2.63$ & 0.115 & \\
\hline \multirow[t]{3}{*}{ ET } & $\leq 29$ & $17(29.31)$ & $7(18.92)$ & 1 & & 0.088 & \multirow[t]{3}{*}{0.048} \\
\hline & $30-36$ & $36(62.07)$ & $21(56.76)$ & 1.417 & $0.51-3.98$ & 0.507 & \\
\hline & $\geq 37$ & $5(8.62)$ & $9(24.32)$ & 4.371 & $1.07-17.79$ & $0.034^{\mathrm{c}}$ & \\
\hline
\end{tabular}

ET, embryo transfer; NC, natural cycle. $p^{\mathrm{f}}$ values were calculated using $\chi^{2}$ trend analysis. ${ }^{\text {a }}$ Comparing 30-36-year-old group with $\geq 37$-year-old group; OR, 3.024; 95\% CI, $1.43-6.41 ; \chi^{2}=8.850, p=0.003$, the difference was statistically significant $(p<0.013) .{ }^{\mathrm{b}} \mathrm{Com}$ paring 30-36-year-old group with $\geq 37$-year-old group; OR, 3.126; 95\% CI, 1.18-8.29, $\chi^{2}=5.603, p=0.018$, the difference was not statistically significant $(p>0.013) .{ }^{c}$ Comparing 30-36-year-old group with $\geq 37$-year-old group; OR, 3.086; 95\% CI, 0.91-10.44, $\chi^{2}=3.469$, $p=0.063$, the difference was not statistically significant $(p>0.013)$.

Table 6. Subgroup analysis of chromosome results in 340 cases stratified by number of previous spontaneous abortions

\begin{tabular}{|c|c|c|c|c|c|c|c|}
\hline Group & $\begin{array}{l}\text { Spontaneous } \\
\text { abortions, } n\end{array}$ & Normal, $n(\%)$ & Abnormal, $n(\%)$ & OR & $95 \% \mathrm{CI}$ & $p$ & $p^{\mathrm{f}}$ \\
\hline \multirow[t]{2}{*}{ Total } & 1 & $42(24.00)$ & $55(33.33)$ & 1 & & 0.017 & 0.002 \\
\hline & $\geq 3$ & $70(50.00)$ & $43(26.06)$ & 0.469 & $0.27-0.82$ & $0.007^{\mathrm{a}}$ & \\
\hline \multirow[t]{2}{*}{$\mathrm{NC}$} & 1 & $19(16.24)$ & $39(30.47)$ & 1 & & 0.006 & 0.001 \\
\hline & 2 & $45(38.46)$ & $53(41.41)$ & 0.574 & $0.29-1.13$ & 0.106 & \\
\hline \multirow{2}{*}{ ET } & 2 & $18(31.03)$ & $14(37.84)$ & 1.118 & $0.43-2.88$ & 0.817 & \\
\hline & $\geq 3$ & $17(29.31)$ & $7(18.92)$ & 0.592 & $0.20-1.76$ & $0.342^{\mathrm{c}}$ & \\
\hline
\end{tabular}

ET, embryo transfer group; NC, natural cycle group; SA, spontaneous abortion. ${ }^{\text {a }}$ Comparing 2-SA group with $\geq 3$-SA group; OR, $0.578 ; 95 \% \mathrm{CI}, 0.35-0.96 ; \chi^{2}=4.437, p=0.035$, the difference was not statistically significant $(p>0.013)$. ${ }^{\mathrm{b}}$ Comparing 2 -SA group with $\geq 3$-SA group; OR, 0.577; 95\% CI, 0.32-1.03; $\chi^{2}=3.475, p=0.062$, the difference was not statistically significant $(p>0.013) .{ }^{c}$ Comparing 2-SA group with $\geq 3$-SA group; OR, $0.529 ; 95 \%$ CI, $0.17-1.63 ; \chi^{2}=1.244, p=0.265$, the difference was not statistically significant $(p>0.013)$.

\section{Discussion}

The incidence of spontaneous abortion is approximately $10 \%$ worldwide [Wilcox et al., 1988]. With delayed parenthood and increasing work pressure over the past few decades, the incidence of spontaneous abortion has increased, and about one-half of the aborted embryos contain chromosomal aberrations [Rai and Regan, 2006]. Although embryonic chromosomal abnormalities are related to maternal age, number of previous miscarriages, and other factors [Ozawa et al., 2019], there are divergent views on the effect of pregnancy achievement methods on embryonic chromosome abnormalities. The purpose of ART is to assist couples in becoming pregnant and having a healthy baby (especially those couples with genetic diseases), and thus genetic testing of embryos is particularly important. The superiority of NGS technology as a means of genetic detection has been increasingly recognized in clinical practice in recent years. The most significant feature of NGS is high throughput, entailing highly parallelized sequencing, which enables thousands of sequencing reactions to be performed simultaneously and producing a large amount of base information from the genome in a single run. NGS is advantageous because it requires less sample and no cell culture, 
and provides whole-genome information, which makes up for the shortcomings of karyotype analysis, FISH, and chip technology. NGS also exhibits a high success rate and accuracy, and has been widely used in tumors, rare genetic diseases, noninvasive prenatal testing, preimplantation genetic testing, and other fields [Ong et al., 2013]. In the next few years, NGS may be also used for disease analysis from single cells as well as DNA in the microcirculation or RNA from blood and/or other body fluids [Ong et al., 2013]. In the present study, we performed a retrospective analysis of the chromosomal karyotypes of 340 patients with early spontaneous abortions using NGS. Earlier studies showed that early spontaneous abortion was related to maternal age and the number of previous miscarriages; however, we observed disparate effects of age and the method of conception on specific chromosomal abnormalities.

According to another study, embryonic chromosome abnormalities were the main cause of spontaneous abortion in early pregnancy, and more than $96 \%$ were numerical chromosome abnormalities [Russo et al., 2016]. Our study indicated that the incidence of chromosomal abnormalities was $48.53 \%$, with aneuploidies accounting for $81.82 \%$ of total abnormalities, and trisomy accounting for $86.67 \%$ of aneuploidy, particularly trisomy 16 . Chromosome 16 accounted for the majority of all chromosomal abnormalities, followed by chromosomes $\mathrm{X}$ and 22 . The distributions and frequencies of trisomy, monosomy, complex abnormalities, and segmental aneuploidies were different (Table 2). At present, it is hypothesized that most aneuploidy cases are caused by nondisjunction or loss of chromosomes in the process of germ cell maturation or in the early cleavage of fertilized eggs. Aneuploidy leads to abortion due to an imbalance in genetic material, which is harmful to embryonic development; however, some aneuploid babies can be delivered, such as those with trisomy 21 or $45, \mathrm{X}$. When chromosomal analysis of early spontaneous abortion was compared with that of blastocyst trophectoderm cells, the trisomy rate increased and the monosomy rate decreased. This suggested that chromosome deletion had a greater impact on embryonic development and implantation relative to duplication [Ozawa et al., 2019], while some gene replication was conducive to embryo implantation [Licciardi et al., 2018]. The lack of genetic material appeared to block some developmental pathways important for early embryo survival and growth, leading to embryonic death [McCallie et al., 2016]. Monosomy primarily occurs during the early stages after embryo implantation [Zhang et al., 2020], which might be equivalent to the biochemical pregnancy stage in the clinic. Abnormal frequencies in the sex chromosomes also increased after fertilization, and previous studies found that abnormal sex chromosomes principally come from the male [Fragouli et al., 2013]. In our study, monosomy was mainly manifested with respect to sex chromosomes, and the probability of chromosomal monosomy was elevated in the period from fertilization to the early stages after embryonic implantation.

The most common autosomal aneuploidy in this study was that of chromosome 16, which is consistent with the majority of previous studies [Fragouli et al., 2013], followed by chromosome 22 . The extra chromosome in trisomy 16 results from nondisjunction of the homologous chromosomes in the first meiotic division, and the proportion of maternal origin is much higher than that of other trisomies [Hassold et al., 1991]. The trisomy 22 incidence was the highest at the blastocyst stage, suggesting that this aneuploidy was more harmful to embryonic implantation and would lead to death, although chromosome 22 is the shortest autosome [Zhang et al., 2020]. In pregnancy or abortion, trisomy 22 predominantly comes from the mother, suggesting that trisomy 22 originating from the male might be fatal; trisomy 22 originating from the mother is usually caused by errors in meiosis [Fragouli et al., 2013]. The abnormalities found in this study did not include chromosome 5 (Table 2), suggesting that chromosome 5 abnormalities might be fatal to embryonic development. Chromosome 5 is longer than chromosomes 16 and 18 and carries more genes that may affect embryonic development or implantation; these abnormalities may then directly result in death [Babariya et al., 2017]. Chromosome 19 has the highest gene density compared with the remaining chromosomes in the human genome, and chromosome 19 abnormalities are also fatal to embryonic development [Zhang et al., 2020]. Our study indicated that the abnormality rate for chromosome 19 was low (Table 2), which further confirms results from previous studies.

A complex abnormality is defined as 2 or more types of abnormalities affecting one or more chromosomes; however, in the present study it primarily signified trisomies involving 2 or more chromosomes. At present, there have been no reports of live births involving 2 or more chromosomal monosomies, because this change is lethal to embryos [Zhang et al., 2020]. Triploidy accounts for approximately $15 \%$ of all chromosomal abnormalities in spontaneous abortions [Levy et al., 2014], and the probability of tetraploids is 1-2\% [Sundvall et al., 2013]. The low incidence of polyploids observed after ICSI is due to the ICSI technology itself, which prevents double-sperm fertilization and entails artificial selection of sperm and oocytes [Martínez et al., 2010; Zhang et al., 2020]. In the present study, polyploids were only triploid (6.66\%), and most of 
these were from natural conceptions. This small percentage may be related to our small sample size and is possibly not an accurate representation of a large population.

Upon further experience with ART, the clinical pregnancy rate has improved significantly, but the clinical delivery rate has remained around $20-30 \%$. Further, most pregnancy losses occur in the first trimester. Extended exposures and high doses of exogenous gonadotropins are experienced during ART [Nagaoka et al., 2012; Sekhon et al., 2017], and in vitro oocyte culture might increase the risk of chromosomal abnormalities in embryos [Perkins et al., 2016]. The aneuploidy risk for conception products was significantly increased in ICSI-assisted pregnancies [Lathi and Milki, 2004] - in particular the incidence of sex chromosome monosomy, which was significantly higher than in a natural-cycle pregnancy [Miao et al., 2009] - and it was speculated to be related to the high risk of sperm chromosome abnormality in infertile males [Lathi and Milki, 2004]. However, some researchers have held different views, proposing that there was no difference in the incidence of chromosomal abnormalities between IVF or ICSI with naturalcycle pregnancy [Miao et al., 2009; Pylyp et al., 2018], and a low risk of chromosomal aneuploidy [Kroon et al., 2011]. The present study thus confirmed that the chromosomal abnormality rate in early pregnancy did not relate to conception methods. However, the chromosome abnormality rate in the NC group (52.24\%) was higher than that in the ET group (38.94\%), which might be related to the selection of gametes and embryos in ART patients; that is, embryos with a higher score - such as blastocysts with higher morphological scores - are transferred first. The embryonic aneuploidy rate at the blastocyst stage was also significantly lower than in cleavage-stage embryos, as some abnormal embryos stop their development before reaching the blastocyst stage [Fragouli et al., 2013]. In our study, sex chromosome abnormalities were the main reason for spontaneous abortions in the ET group, which was related to artificial selection during IVF; however, the specific mechanism remains unclear. Although the aneuploidy rate was the highest in both the ET and NC groups, the distribution of abnormal chromosomes in the 2 groups was completely different (Table 3), except for the difference in the sample size of the 2 groups; this finding may be related to the different conception methods.

Most investigators opine that advanced age is a risk factor for embryonic chromosomal abnormalities. The rate of chromosomal anomalies increases significantly with age [Grande et al., 2012], particularly the aneuploidy rate [Kroon et al., 2011; Fragouli et al., 2013]. In ART, the pregnancy rate in older women was low, but they maintained a similar live-birth rate compared to young women after receiving donated eggs [Luke et al., 2012], thus suggesting that age decreases live-birth rates due to poor oocyte quality. Over $90 \%$ of the chromosomal imbalances were of maternal origin due to premature separation of sister chromatids in meiosis [Nagaoka et al., 2012; Cimadomo et al., 2018], with the most common separation mode being reverse separation [Ottolini et al., 2015]. Aging oocytes exhibited a diminished fertilization rate, thus resulting in subsequent embryonic developmental aberrations due to mitochondrial dysfunction, included shortened telomeres, adhesin dysfunction, and damage to the spindle assembly checkpoint [Miao et al., 2009; Keefe et al., 2015]. In the current study, we showed that with increasing age, the risk of chromosomal aberrations increased (Table 3), which is consistent with a study by Grande et al. [2012]. In our study, there was a significant difference in the incidence of chromosomal abnormalities between the $\geq 37$-year group and the $\leq 29$-year group and between the 30-36-year group and the $\geq 37$-year group. The relationship between age and abnormal chromosomes in the NC and ET groups also showed a similar trend (Table 3 ). However, low-frequency chromosomal abnormalities were different in each age group (Table 2). For example, $\mathrm{X}$ chromosome abnormalities were more common in the $\leq 29$-year group, but complex abnormality rates were lower. In the 30-36-year group, chromosome 16 abnormalities were more common, but segmental aneuploidy was lower. In the $\geq 37$-year group, chromosome 22 abnormalities were more common, but segmental aneuploidies were not detected. Overall, with increasing age, the abnormality rates in chromosomes 12,15 , 20 , and 22 increased, while those for chromosomes $6,7,13$, and $\mathrm{X}$ decreased, indicating that not all chromosomal abnormalities increased with age. For abnormal types, monosomy incidence decreased with age $(19.23 \%$ vs. $9.76 \%$ vs. $0.00 \%)$, while trisomy incidence increased $(55.77 \%$ vs. $74.39 \%$ vs. $87.10 \%$ ), confirming the correlation between trisomy and advanced age as well as the correlation between monosomy and younger patients [Hagman et al., 2010]. Additionally, the correlation between advanced reproductive age and trisomy was not affected by other clinical factors such as the number of previous miscarriages or fetal size [Ozawa et al., 2019]. The incidence of other chromosomal abnormalities - such as haploidy, triploidy, complex abnormalities, segmental aneuploidies, microdeletion, and microduplication - were also independent of age. Intriguingly, a comparison of sperm chromosomal aberrations between patients that were 59-74 years old and 23-39 years old indicated that older men had a higher risk of chromosomal aberration, resulting in chromosomal abnormal- 
ities in their offspring [Sartorelli et al., 2001]. Consequently, the male's age should also be considered in genetic counseling for infertile couples. For patients with recurrent miscarriage, Ozawa et al. [2019] indicated that the rate of chromosomal abnormalities of conception products decreased with previous miscarriages, suggesting that the likelihood of producing a normal embryo increased with the number of previous miscarriages. The current study also showed that as the number of previous miscarriages increased, the risk of chromosomal abnormalities was attenuated (Table 6). This difference was statistically significant in the group with 1 previous spontaneous abortion relative to $\geq 3$ previous spontaneous abortions, which further validated Ozawa's view [Ozawa et al., 2019]. This indicated that the risk for spontaneous abortion caused by nonembryonic chromosomal abnormalities increased with the elevation in abortion frequency. However, a similar phenomenon was not detected in the ET group, possibly due to artificial intervention during treatment with ART.

There are many chromosomal CNVs in the human genome, and these constitute the basis for evolution, genetic diversity, and disease susceptibility [Neofytou et al., 2017]. The incidence of pathogenic micro-CNVs in abortion is 0.6-0.78\% [Levy et al., 2014; Sahoo et al., 2017], but it is uncertain whether these micro-CNVs lead to abortion. In the present study, there were 6 cases of segmental aneuploidy deletions (containing 2 micro-CNVs) and 3 cases of segmental aneuploidy duplications, accounting for $5.45 \%$ of abnormal cases.

Villous chromosomal detection is of immense significance in the analysis of the causes of miscarriage, and NGS can provide more details as to cytogenetic information of embryos. Our study confirmed that chromosomal abnormalities in villi are the primary cause of early spontaneous abortion, and we found these anomalies to be related to age and the number of previous miscarriages. However, due to our relatively small sample size, we could not elucidate in detail the changing trends in specific chromosomal abnormalities with age, nor fully determine the relationship between methods of conception and embryonic chromosomal abnormalities. Thus, it is still necessary to examine the factors related to chromosomal abnormalities using a larger sample size in future studies.

\section{Conclusion}

In the present study, we found that chromosomal abnormality was a dominant cause of pregnancy loss in the first trimester. Maternal age and number of previous miscarriages were closely related to chromosomal aberration, but conception methods, number of pregnancies, deliveries, or induced abortions were not correlated with the occurrence of chromosomal aberrations. And, importantly, not all chromosomal abnormalities increased with age. The type and distribution of low-frequency abnormal chromosomes was different between the EC and NC groups. However, whether different conception methods such as ART affect the occurrence or rate of some chromosomal abnormalities requires further exploration.

\section{Acknowledgements}

The authors thank both the patients and laboratory colleagues who provided us with the experiences necessary to develop this study. We would like to thank LetPub (www.letpub.com) for providing linguistic assistance during the preparation of this manuscript.

\section{Statement of Ethics}

This study was approved by the Ethics Committee of Shanxi Maternal and Child Health Care Hospital (IRB-KY-2020). All patients provided signed informed consent for genetic analysis of villi by NGS.

\section{Conflict of Interest Statement}

The authors have no conflicts of interest to declare.

\section{Funding Sources}

This work was supported by the National Key Research and Development Program (2018YFC1002103) and the Hospital Fund of Shanxi Maternal and Child Health Care Hospital (201727 and 202008).

\section{Author Contributions}

Xueluo Zhang, Junmei Fan, and Yanhua Chen contributed to data collection and interpretation as well as writing of the manuscript; Zhijiao Song contributed to the analysis of the data; Jun Wang, Jinghui Zhao, and Zhongyun Li contributed to data collection and collation; and Xueqing $\mathrm{Wu}$ and Yuanjing $\mathrm{Hu}$ contributed to the concept and design of the study. All authors approved the final version before publication. 


\section{References}

Angiolucci M, Murru R, Melis G, Carcassi C, Mais $\mathrm{V}$. Association between different morphological types and abnormal karyotypes in early pregnancy loss. Ultrasound Obstet Gynecol. 2011;37(2):219-25.

Babariya D, Fragouli E, Alfarawati S, Spath K, Wells D. The incidence and origin of segmental aneuploidy in human oocytes and preimplantation embryos. Hum Reprod. 2017; 32(12):1-12.

Bauer J. The Molecular Revolution in Cutaneous Biology: Era of Cytogenetics and Copy Number Analysis. J Invest Dermatol. 2017;137(5): e57-9.

Capalbo A, Hoffmann ER, Cimadomo D, Maria Ubaldi F, Rienzi L. Human female meiosis revised: new insights into the mechanisms of chromosome segregation and aneuploidies from advanced genomics and time-lapse imaging. Hum Reprod Update. 2017;23(6):70622.

Cimadomo D, Fabozzi G, Vaiarelli A, Ubaldi N, Ubaldi FM, Rienzi L. Impact of Maternal Age on Oocyte and Embryo Competence. Front Endocrinol (Lausanne). 2018;9:327.

Dong Z, Zhang J, Hu P, Chen H, Xu J, Tian Q, et al. Low-pass whole-genome sequencing in clinical cytogenetics: a validated approach. Genet Med. 2016;18(9):940-8.

Doubilet PM, Benson CB, Bourne T, Blaivas M, Society of Radiologists in Ultrasound Multispecialty Panel on Early First Trimester Diagnosis of Miscarriage and Exclusion of a Viable Intrauterine Pregnancy, Barnhart KT, et al. Diagnostic criteria for nonviable pregnancy early in the first trimester. N Engl J Med. 2013; 369(15):1443-51.

Fragouli E, Alfarawati S, Spath K, Jaroudi S, Sarasa J, Enciso M, et al. The origin and impact of embryonic aneuploidy. Hum Genet. 2013; 132(9):1001-13.

Grande M, Borrell A, Garcia-Posada R, Borobio V, Muñoz M, Creus M, et al. The effect of maternal age on chromosomal anomaly rate and spectrum in recurrent miscarriage. Hum Reprod. 2012;27(10):3109-17.

Hagman A, Wennerholm UB, Källén K, Barrenäs ML, Landin-Wilhelmsen K, Hanson C, et al. Women who gave birth to girls with Turner syndrome: maternal and neonatal characteristics. Hum Reprod. 2010;25(6):1553-60.

Hassold TJ, Pettay D, Freeman SB, Grantham M, Takaesu N. Molecular studies of non-disjunction in trisomy 16. J Med Genet. 1991;28(3): 159-62.

Jobanputra V, Sobrino A, Kinney A, Kline J, Warburton D. Multiplex interphase FISH as a screen for common aneuploidies in spontaneous abortions. Hum Reprod. 2002;17(5): 1166-70.

Keefe D, Kumar M, Kalmbach K. Oocyte competency is the key to embryo potential. Fertil Steril. 2015;103(2):317-22.
Kroon B, Harrison K, Martin N, Wong B, Yazdani A. Miscarriage karyotype and its relationship with maternal body mass index, age, and mode of conception. Fertil Steril. 2011; 95(5):1827-9.

Lathi RB, Milki AA. Rate of aneuploidy in miscarriages following in vitro fertilization and intracytoplasmic sperm injection. Fertil Steril. 2004;81(5):1270-2.

Levy B, Sigurjonsson S, Pettersen B, Maisenbacher MK, Hall MP, Demko Z, et al. Genomic imbalance in products of conception: singlenucleotide polymorphism chromosomal microarray analysis. Obstet Gynecol. 2014;124(2 Pt 1):202-9.

Licciardi F, Lhakhang T, Kramer YG, Zhang Y, Heguy A, Tsirigos A. Human blastocysts of normal and abnormal karyotypes display distinct transcriptome profiles. Sci Rep. 2018; 8(1):14906.

Luke B, Brown MB, Wantman E, Lederman A, Gibbons W, Schattman GL, et al. Cumulative birth rates with linked assisted reproductive technology cycles. N Engl J Med. 2012; 366(26):2483-91.

Martínez MC, Méndez C, Ferro J, Nicolás M, Serra V, Landeras J. Cytogenetic analysis of early nonviable pregnancies after assisted reproduction treatment. Fertil Steril. 2010;93(1): 289-92.

McCallie BR, Parks JC, Patton AL, Griffin DK, Schoolcraft WB, Katz-Jaffe MG. Hypomethylation and Genetic Instability in Monosomy Blastocysts May Contribute to Decreased Implantation Potential. PLoS One. 2016;11(7): e0159507.

Miao YL, Kikuchi K, Sun QY, Schatten H. Oocyte aging: cellular and molecular changes, developmental potential and reversal possibility. Hum Reprod Update. 2009;15(5):573-85.

Nagaoka SI, Hassold TJ, Hunt PA. Human aneuploidy: mechanisms and new insights into an age-old problem. Nat Rev Genet. 2012;13(7): 493-504.

Neofytou MC, Tsangaras K, Kypri E, Loizides C, Ioannides M, Achilleos A, et al. Targeted capture enrichment assay for non-invasive prenatal testing of large and small size sub-chromosomal deletions and duplications. PLoS One. 2017;12(2):e0171319.

Ong FS, Lin JC, Das K, Grosu DS, Fan JB. Translational utility of next-generation sequencing. Genomics. 2013;102(3):137-9.

Ottolini CS, Newnham L, Capalbo A, Natesan SA, Joshi HA, Cimadomo D, et al. Genome-wide maps of recombination and chromosome segregation in human oocytes and embryos show selection for maternal recombination rates. Nat Genet. 2015;47(7):727-35.

Ouyang Y, Tan Y, Yi Y, Gong F, Lin G, Li X, et al. Correlation between chromosomal distribution and embryonic findings on ultrasound in early pregnancy loss after IVF-embryo transfer. Hum Reprod. 2016;31(10): 2212-8.
Ozawa N, Ogawa K, Sasaki A, Mitsui M, Wada S, Sago H. Maternal age, history of miscarriage, and embryonic/fetal size are associated with cytogenetic results of spontaneous early miscarriages. J Assist Reprod Genet. 2019;36(4): 749-57.

Perkins AT, Das TM, Panzera LC, Bickel SE. Oxidative stress in oocytes during midprophase induces premature loss of cohesion and chromosome segregation errors. Proc Natl Acad Sci USA. 2016;113(44):E6823-30.

Pylyp LY, Spynenko LO, Verhoglyad NV, Mishenko AO, Mykytenko DO, Zukin VD. Chromosomal abnormalities in products of conception of first-trimester miscarriages detected by conventional cytogenetic analysis: a review of 1000 cases. J Assist Reprod Genet. 2018;35(2):265-71.

Rai R, Regan L. Recurrent miscarriage. Lancet. 2006;368(9535):601-11.

Russo R, Sessa AM, Fumo R, Gaeta S. Chromosomal anomalies in early spontaneous abortions: interphase FISH analysis on 855 FFPE first trimester abortions. Prenat Diagn. 2016; 36(2):186-91.

Sahoo T, Dzidic N, Strecker MN, Commander S, Travis MK, Doherty C, et al. Comprehensive genetic analysis of pregnancy loss by chromosomal microarrays: outcomes, benefits, and challenges. Genet Med. 2017;19(1):83-9.

Sartorelli EM, Mazzucatto LF, de Pina-Neto JM. Effect of paternal age on human sperm chromosomes. Fertil Steril. 2001;76(6):1119-23.

Sekhon L, Shaia K, Santistevan A, Cohn KH, Lee JA, Beim PY, et al. The cumulative dose of gonadotropins used for controlled ovarian stimulation does not influence the odds of embryonic aneuploidy in patients with normal ovarian response. J Assist Reprod Genet. 2017;34(6):749-58.

Shah MS, Cinnioglu C, Maisenbacher M, Comstock I, Kort J, Lathi RB. Comparison of cytogenetics and molecular karyotyping for chromosome testing of miscarriage specimens. Fertil Steril. 2017;107(4):1028-33.

Sugiura-Ogasawara M, Ozaki Y, Katano K, Suzumori N, Kitaori T, Mizutani E. Abnormal embryonic karyotype is the most frequent cause of recurrent miscarriage. Hum Reprod. 2012; 27(8):2297-303.

Sundvall L, Lund H, Niemann I, Jensen UB, Bolund L, Sunde L. Tetraploidy in hydatidiform moles. Hum Reprod. 2013;28(7):2010-20.

Wilcox AJ, Weinberg CR, O'Connor JF, Baird DD, Schlatterer JP, Canfield RE, et al. Incidence of early loss of pregnancy. N Engl J Med. 1988;319(4):189-94.

Winter E, Wang J, Davies MJ, Norman R. Early pregnancy loss following assisted reproductive technology treatment. Hum Reprod. 2002;17(12):3220-3.

Zhang X, Wang Y, Zhao N, Liu P, Huang J. Variations in chromosomal aneuploidy rates in IVF blastocysts and early spontaneous abortion chorionic villi. J Assist Reprod Genet. 2020;37(3):527-37. 